\title{
Self-Reported Alcohol, Tobacco, and Cannabis Use during COVID-19 Lockdown Measures: Results from a Web-Based Survey
}

\author{
Nathalie Vanderbruggen ${ }^{a}$ Frieda Matthys $^{a} \quad$ Sven Van Laere $^{b}$ Dieter Zeeuws ${ }^{a}$ \\ Liesbeth Santermans $^{a} \quad$ Seline Van den Ameele ${ }^{a} \quad$ Cleo L. Crunelle ${ }^{a, c}$ \\ aDepartment of Psychiatry, Vrije Universiteit Brussel (VUB), Universitair Ziekenhuis Brussel (UZ Brussel), Brussels, \\ Belgium; ' Interfaculty Center Data Processing \& Statistics, Vrije Universiteit Brussel (VUB), Brussels, Belgium; \\ 'Toxicological Center, Antwerp University, Antwerp, Belgium
}

\section{Keywords}

Alcohol - Nicotine - Cannabis - Coronavirus disease 19.

Survey $\cdot$ Motives $\cdot$ Substance use

\begin{abstract}
Background: The outbreak of coronavirus disease 19 (COVID-19) has led to measures of social distancing and quarantine worldwide. This stressful period may lead to psychological problems, including increases in substance use. Objective: To investigate changes in alcohol, tobacco, and cannabis consumption before and during COVID-19 lockdown and motives for these changes in substance use. Method: A web-based survey was filled out by an unselected population during the social distancing measures of the $\mathrm{CO}-$ VID-19 pandemic in Belgium that assessed changes in alcohol, tobacco, and cannabis consumption in the period before and during the COVID-19 lockdown and also asked about reasons for change. Results: A total of 3,632 respondents (mean age $42.1 \pm 14.6$ years; $70 \%$ female) filled out the survey. Overall, respondents reported consuming more alcohol $(d=0.21)$ and smoking more cigarettes $(d=0.13)$ than before the COVID-19 pandemic (both $p<0.001$ ), while no significant changes in the consumption of cannabis were noted. The odds of consuming more alcohol during the lock-
\end{abstract}

karger@karger.com

(c) 2020 S. Karger AG, Basel

www.karger.com/ear

Karger down were associated with younger age $(\mathrm{OR}=0.981, p<$ 0.001 ), more children at home ( $\mathrm{OR}=1.220, p<0.001)$, nonhealthcare workers $(p<0.001)$, and being technically unemployed related to COVID-19 $(p=0.037)$. The odds of smoking more cigarettes during the lockdown were associated with younger age ( $O R=0.988, p=0.027)$, current living situation $(p<0.001)$, lower education $(p=0.015)$, and working situation related to COVID-19 $(p=0.018)$. Boredom, lack of social contacts, loss of daily structure, reward after a hard-working day, loneliness, and conviviality were the main reasons for consuming more of the various substances. Conclusions: During the lockdown, individuals consumed slightly more alcohol and smoked marginally more cigarettes compared to the period before the lockdown. Further research focussing on follow-up of individuals at risk may be useful to provide appropriate care in post-COVID times.

(c) 2020 S. Karger AG, Basel

\section{Introduction}

COVID-19 (coronavirus disease 2019) is an infectious disease caused by the virus SARS-CoV-2. The infection is highly contagious and causes severe pneumonia, acute respiratory distress syndrome, and death particularly in 
vulnerable populations including older adults and people with chronic medical conditions such as cardiovascular disease, diabetes, respiratory disease, hypertension, and malignancy [1].

In an attempt to control the COVID-19 pandemic, physical distancing and hygiene measures, including regular hand washing and covering of nose and mouth when sneezing or coughing, were implemented worldwide. In several countries, sudden and dramatic social changes were implemented to slow contagion and "flatten the curve" of the pandemic to preserve capacity of healthcare resources, which included asking people to stay at home as much as possible [2].

Quarantine or social isolation is stressful and associated with psychological problems, especially in older adults, females, individuals from lower socio-economic groups, and those with underlying mental health conditions $[3,4]$. Psychological problems can include anxiety, depression, acute stress disorder, substance abuse, insomnia, and post-traumatic stress disorder $[3,5]$.

A review on the impact of the major infectious disease outbreak of SARS in 2003 shows that the psychological impact is mainly associated with occupational role, high-risk work environment, quarantine, social support, and the impact on our personal and professional life [6]. Having been quarantined and working in hospital or wards during the 2003 SARS outbreak were linked with the development of alcohol abuse and dependence symptoms 3 years later [7]. Drinking was self-reported by hospital employees as a coping method and was found significantly related with hyperarousal [7]. The main predictors of alcohol consumption after a massive traumatic experience were alcohol consumption before the event and the rationale for drinking during the event, where drinking as a coping mechanism and drinking for pleasure were the main predictors for long-term alcohol consumption up to 10 years later [8].

The almost global lockdown is unique, and little is known about the consumption of substances during social isolation. We hypothesized that individuals in social isolation due to lockdown measures would consume more substances. Mapping the changes in substance use and the reasons for these changes during large-scale general social isolation is important. It reveals the response of the general population in times of stress and may, therefore, be of valuable help to provide appropriate care in post-COVID times and indicate possible preventive actions in case of similar events. In this study, we report on changes in alcohol, tobacco, and cannabis use in the midst of the COVID-19 lockdown in Belgium and report on the major motives for the change in consumption.

\section{Materials and Methods}

\section{Study Population and Data Collection}

A cross-sectional survey was sent out over a brief period of time (20 days; between April 9, 2020, and April 29, 2020) during the full COVID-19 lockdown measures in Belgium. The survey was distributed by the communication services of the University Hospital of Brussels (UZ Brussel) and the university (Vrije Universiteit Brussel) and was circulated through online social media (Facebook and Twitter). The target population included adult Belgian residents, irrespective of age, living area, or current substance use. To reach out to an unselected population, the survey was made available by a link to the survey on Qualtrics online and the link was shared through social media, including Facebook (targeting a broad range of individuals), the websites of the university (targeting students) and the university hospital (targeting patients and hospital co-workers), and the website SeniorenNet.be (targeting the senior generation).

Individuals older than 18 years and living or residing in Belgium during the COVID-19 lockdown measures were asked to fill in the survey online. There were no formal exclusion criteria. The ethics committee of the University Hospital Brussels, Belgium, approved this study on April 9, 2020 (No. 143202043).

\section{Measures}

The questionnaire consisted of 5 different parts with 20 questions regarding (1) demographics, (2) smoking cigarettes, (3) alcohol use, (4) cannabis use, and (5) factors that influenced substance use during the COVID-19 lockdown measures. The questionnaire was made available in Dutch, French, and English.

Demographic questions included age, gender, and postal code. Age was asked as an age category (per 5 years). Postal code was recoded into the variables "urban" and "rural." Current living situation (with partner, with children, with others, or alone), social situation (age and number of children and cared for at home or outside of home), and professional situation (profession, education, and currently home-working) were also asked.

Smoking prior to the lockdown measures was noted as the number of cigarettes smoked on an average day in the period before the COVID-19 lockdown. Response options included "not smoking" (0 cigarettes per day), smoking 1-5 cigarettes per day, smoking 6-10 cigarettes per day, smoking 11-19 cigarettes per day, or smoking 20 or more cigarettes per day. Smoking during the current COVID-19 lockdown was assessed as the average number of cigarettes smoked now and by asking whether they currently smoke more, less, or about the same compared to before the lockdown. In case they smoked more or less, they were questioned about the average number of additional or fewer cigarettes on a normal day, using the same response options regarding smoking prior to the lockdown measures.

For questions regarding alcohol use, reference was made to alcohol units per day. A standard drink (one alcohol unit) was defined as $10 \mathrm{~g}$ of pure alcohol. This corresponds to one $25 \mathrm{cL}$ glass of beer ( $5 \%$ alcohol), $10 \mathrm{cL}$ of wine ( $12 \%$ alcohol), $5 \mathrm{cL}$ of aperitif ( $15 \%$ alcohol), or $3.5 \mathrm{cL}$ of distilled spirits (35\% alcohol). Respondents were asked how much alcohol he/she drank on an average day before the COVID-19 measures and how much he/she is drinking now (during the lockdown measures), with additionally specifying whether he/she drank more, less, or about the same than before. Response options referred to the average quantity of alco- 
hol consumption during the prior week (rather than the frequency) and included the options "I do not drink alcohol" (mean of 0 alcohol units per day) and "on average 1 alcohol unit per day" to "on average $>12$ alcohol units per day," with specifically denoting whether they drank more, less, or about the same as usual.

Cannabis use was assessed as mean amount of cannabis joints smoked per day, both before and during the COVID-19 lockdown measures. The questions concerned the average amount of cannabis consumption per day and specifically asked whether the respondent smoked less, more, or about the same.

The final question asked about motives for increased use of any substance during the lockdown with several options (listed further in Table 4). Those options were not mutually exclusive; several options could be chosen together, and there was opportunity for personal additions from the respondents, as "others, please specify:_.."

\section{Statistical Analyses}

In the survey, age, amount of alcohol units, smoking cigarettes, and cannabis use were recorded in categories. For making these outcomes linear, we applied a transformation of the outcomes by using the mean value of the upper and the lower bound of the categories. Profession was recoded taking into account the variables "do you work in healthcare" and "profession," thereby regrouping free text into 6 categories: working healthcare, working nonhealthcare, student, retired, job seeking, and others.

Linear variables were presented as mean \pm SD where applicable. Categorical variables were reported using the number of observations and the percentage of observations. Comparison between the amounts of use in both periods (before and during the COVID-19 lockdown measures) was performed using a Wilcoxon signed-rank test. Cohen's $d$ were calculated and represent the effect size. McNemar tests were used to compare substance use before and during the lockdown. In these McNemar tests, a categorization of "using more" or "using less or equal" was used. When $>5 \%$ of the respondents showed to consume more during the lockdown compared to before, a binary logistic regression was executed. Here, a backwards regression model building technique was used with variables (age, sex, living in a city, family situation, number of children, professional occupation, educational background, and whether or not you work more from home during the lockdown). Removal of a predictor variable or factor was considered appropriate when having a significance of $>0.10$. Furthermore, a significance level of 0.05 was considered statistically significant for all tests. In order to examine the motives for substance use and the reason why respondents were using more during the COVID-19 measures than before, a descriptive analysis was executed. After reporting the frequencies, the motives were ranked from highest present motive to lowest present motive for each of the substances. Statistics were performed using SPSS version 26.

\section{Results}

\section{Respondents' Characteristics}

A total of 4,098 respondents participated, with 3,632 (89\%) completing the survey. The mean age was $42.1 \pm$ 14.6 years. The characteristics of the $n=3,632$ respon-
Table 1. Characteristics of respondents

\begin{tabular}{|c|c|c|}
\hline & \multicolumn{2}{|c|}{$\begin{array}{l}\text { Study respondents } \\
\text { (total } N=3,632)\end{array}$} \\
\hline & $N$ & $\%$ \\
\hline Age, years $($ mean $\pm S D)$ & \multicolumn{2}{|c|}{$42.1 \pm 14.6$} \\
\hline \multicolumn{3}{|l|}{ Gender } \\
\hline Female & 2,541 & 70.0 \\
\hline Male & 1,083 & 29.8 \\
\hline Gender-neutral & 8 & 0.2 \\
\hline \multicolumn{3}{|l|}{ Language used to fill in survey } \\
\hline Dutch & 3,361 & 92.5 \\
\hline French & 200 & 5.5 \\
\hline English & 71 & 2.0 \\
\hline \multicolumn{3}{|l|}{ Area of residence } \\
\hline Urban & 865 & 23.8 \\
\hline Rural & 2,767 & 76.2 \\
\hline \multicolumn{3}{|l|}{ Profession } \\
\hline In healthcare & 1,370 & 36.8 \\
\hline Not in healthcare & 2,295 & 63.2 \\
\hline \multicolumn{3}{|l|}{ Educational background } \\
\hline Primary education & 28 & 0.8 \\
\hline Vocational education & 72 & 2.0 \\
\hline Secondary education & 562 & 15.5 \\
\hline Higher education & 2,762 & 76.0 \\
\hline Doctorate & 189 & 5.2 \\
\hline Missing & 19 & 0.5 \\
\hline \multicolumn{3}{|l|}{ Current living situation } \\
\hline Living alone & 556 & 15.3 \\
\hline Living with partner and/or children & 2,500 & 68.8 \\
\hline With children $0-5$ years old & 525 & $21.0^{*}$ \\
\hline With children $6-12$ years old & 550 & $22.0^{*}$ \\
\hline With children $13-18$ years old & 457 & $18.3^{*}$ \\
\hline With children older than 18 years & 565 & $22.6^{*}$ \\
\hline Living with others & 576 & 15.9 \\
\hline Household with children & 1,609 & 44.3 \\
\hline Cared for at home & 1,358 & 37.4 \\
\hline Cared for outside of home & 190 & 5.2 \\
\hline Part of the children cared for at home & 61 & 1.7 \\
\hline
\end{tabular}

Characteristics of respondents $(N=3,632)$ in the Belgian ALCOVID study. SD, standard deviation. * Percentage of respondents living with partner and/or children.

dents are specified in Table 1. Most respondents were female (70\%), between 23 and 47 years old (70\%), mostly from rural areas $(76 \%)$, with higher education $(78 \%)$, and living with a partner $(69 \%)$. The vast majority of the participants (93\%) filled out the questionnaire in Dutch.

\section{Alcohol Use}

2,243 respondents $(61.8 \%)$ were consuming alcohol before the lockdown. Of these, 341 quit drinking during the lockdown (9.4\% of the total sample). Of the 1,389 
Table 2. Logistic regression regarding alcohol use

\begin{tabular}{|c|c|c|c|c|}
\hline & \multirow[t]{2}{*}{ OR } & \multicolumn{2}{|c|}{$95 \% \mathrm{CI}$ for OR } & \multirow[t]{2}{*}{$p$ value } \\
\hline & & lower & upper & \\
\hline Age, years & 0.981 & 0.974 & 0.987 & $<0.001$ \\
\hline Children, $n$ & 1.220 & 1.146 & 1.298 & $<0.001$ \\
\hline Are you more at home during the lockdown? & & & & 0.037 \\
\hline Yes, with tele-work & Ref. & & & - \\
\hline Yes, due to technical unemployment & 1.357 & 1.065 & 1.728 & 0.013 \\
\hline Yes, due to illness & 0.842 & 0.541 & 1.311 & 0.447 \\
\hline Yes, due to illness related to COVID-19 & 0.813 & 0.434 & 1.526 & 0.520 \\
\hline No & 0.882 & 0.727 & 1.069 & 0.201 \\
\hline Professional occupation & & & & $<\mathbf{0 . 0 0 1}$ \\
\hline Healthcare worker & Ref. & & & - \\
\hline Non-healthcare worker & 1.404 & 1.176 & 1.676 & $<0.001$ \\
\hline Student & 0.537 & 0.389 & 0.740 & $<0.001$ \\
\hline Job seeker & 1.086 & 0.468 & 2.521 & 0.848 \\
\hline Retired & 1.312 & 0.879 & 1.957 & 0.184 \\
\hline Others (e.g., disabled person) & 0.810 & 0.40 & 1.527 & 0.516 \\
\hline
\end{tabular}

Logistic regression explaining relationships to why respondents consumed more alcohol during the lockdown compared to before. In total, 1,102 respondents out of 3,632 (30.3\%) noted drinking more during the lockdown compared to before.

non-consuming respondents before the lockdown, 211 started consuming alcohol during the lockdown (5.8\% of the total sample; $p<0.001$ ). Of all respondents, $30.3 \%$ stated that they drank more and $13.7 \%$ stated to drink less during than before the lockdown. A statistically significant, but small $(d=0.21)$, difference was found between the number of drinks per day before and during the lockdown $(1.0 \pm 1.4$, range $0-15$, and $1.4 \pm 2.1$, range $0-21$, respectively; $p<0.001)$.

Logistic regression analysis (see Table 2 ) shows that the odds of consuming more alcohol decreased during the lockdown with $1.9 \%$ on average per age year of the respondent ( $\mathrm{OR}=0.981 ; p<0.001$ ). Also, a higher number of children at home increased the odds of consuming more alcohol during lockdown measures $(p<0.001)$ : per extra child in the family, the odds increased with $22 \%$ $(\mathrm{OR}=1.220$; 95\% CI: 1.146-1.298). A 3rd variable of influence was whether a respondent now works more from home compared to before the lockdown $(p=0.037)$. Technical unemployed respondents are $36 \%$ more likely to drink more $(\mathrm{OR}=1.357 ; p=0.013)$ compared to respondents at home due to tele-working. Also, the professional occupation of the respondent played a role in drinking more during the lockdown $(p<0.001)$, with non-healthcare workers being $40 \%$ more likely to drink compared to healthcare workers during the lockdown $(\mathrm{OR}=1.404 ; p<0.001)$. Students, however, were on aver- age $46 \%$ less likely to drink more compared to healthcare workers $(\mathrm{OR}=0.537 ; p<0.001)$. Gender, living in a city, family situation, and educational background were variables without significant effect on the change in alcohol use.

\section{Cigarettes}

558 respondents (15.4\%) smoked cigarettes before the lockdown. Of these, 36 quit smoking during the lockdown (1.0\% of the total sample). Of the 3,074 non-smokers before the lockdown, 34 started smoking during the lockdown ( $0.9 \%$ of the total sample). Of all respondents, $7.4 \%$ stated smoking more and $2.5 \%$ stated smoking less during than before the lockdown. A statistically significant, but negligible $(d=0.13)$, difference was found between the number of cigarettes per day before and during the lockdown $(1.5 \pm 4.5$, range $0-30$, and $1.7 \pm 5.5$, range $0-60$, respectively; $p<0.001)$.

Table 3 shows the results from the logistic regression analysis. Age was inversely associated with the odds of smoking cigarettes being $12 \%$ per 10 years more during than before the lockdown ( $\mathrm{OR}=0.988 ; p=0.027)$. Also, living situation had a statistically significant relationship with increased smoking during the lockdown $(p<0.001)$ : living alone more than doubled the odds of smoking more cigarettes $(\mathrm{OR}=2.159$; 95\% CI: $1.587-2.938)$ during the lockdown compared to living together with a partner and/or child. Educational background also had a sig- 
Table 3. Logistic regression regarding nicotine use

\begin{tabular}{|c|c|c|c|c|}
\hline & \multirow[t]{2}{*}{ OR } & \multicolumn{2}{|c|}{$95 \% \mathrm{CI}$ for OR } & \multirow[t]{2}{*}{$p$ value } \\
\hline & & lower & upper & \\
\hline Age, years & 0.988 & 0.978 & 0.999 & 0.027 \\
\hline Current living situation & & & & $<0.001$ \\
\hline Living with partner and/or child & Ref. & & & - \\
\hline Living alone & 2.159 & 1.587 & 2.938 & $<0.001$ \\
\hline Living with others & 1.081 & 0.739 & 1.580 & 0.690 \\
\hline Educational background & & & & 0.015 \\
\hline Higher education & Ref. & & & - \\
\hline Primary education & 1.114 & 0.258 & 4.817 & 0.885 \\
\hline Vocational education & 2.085 & 1.025 & 4.240 & 0.042 \\
\hline Secondary education & 1.312 & 0.944 & 1.823 & 0.106 \\
\hline Doctorate & 0.245 & 0.077 & 0.777 & 0.017 \\
\hline Do you work more at home now? & & & & 0.018 \\
\hline Yes, with tele-work & Ref. & & & \\
\hline Yes, due to technical unemployment & 1.638 & 1.125 & 2.386 & 0.010 \\
\hline Yes, due to illness & 1.431 & 0.758 & 2.702 & 0.269 \\
\hline Yes, due to illness related to COVID-19 & 1.069 & 0.413 & 2.766 & 0.890 \\
\hline No & 0.820 & 0.598 & 1.123 & 0.260 \\
\hline
\end{tabular}

Logistic regression explaining relationships to why respondents smoke more cigarettes during the lockdown compared to before. In total, 264 respondents out of 3,632 (7.3\%) noted they smoked more during the lockdown compared to before.

Table 4. Ranking of motives for smoking more, drinking more, and using more cannabis

\begin{tabular}{|c|c|c|c|c|c|c|c|c|c|}
\hline \multirow[t]{2}{*}{ Reason for more consuming of... } & \multicolumn{3}{|c|}{ Nicotine } & \multicolumn{3}{|c|}{ Alcohol } & \multicolumn{3}{|c|}{ Cannabis } \\
\hline & $N$ & $\%$ & rank & $N$ & $\%$ & rank & $N$ & $\%$ & rank \\
\hline Has nothing to do with lockdown & 16 & 6.1 & 12 & 26 & 2.4 & 13 & 2 & 2.6 & 12 \\
\hline Fear of contamination & 10 & 3.8 & 14 & 35 & 3.2 & 12 & 2 & 2.6 & 12 \\
\hline Reward after a hard-working day & 98 & 37.1 & 3 & 513 & 46.6 & 2 & 33 & 43.4 & 3 \\
\hline Increased tension (family, work, etc.) & 59 & 22.3 & 7 & 270 & 24.5 & 6 & 17 & 22.4 & 8 \\
\hline Decreased tension (traffic, more sleep time, etc.) & 27 & 10.2 & 11 & 123 & 11.2 & 11 & 16 & 21.1 & 9 \\
\hline Stress from negative reporting & 50 & 18.9 & 8 & 70 & 15.4 & 8 & 19 & 25.0 & 7 \\
\hline Loneliness & 75 & 28.4 & 5 & 168 & 15.2 & 9 & 25 & 32.9 & 5 \\
\hline Lack of social contacts & 108 & 40.9 & 2 & 383 & 34.8 & 3 & 37 & 48.7 & 2 \\
\hline Lack of activities or sports & 46 & 17.4 & 9 & 194 & 17.6 & 7 & 22 & 28.9 & 6 \\
\hline More tension due to permanent presence of housemates & 38 & 14.4 & 10 & 157 & 14.2 & 10 & 9 & 11.8 & 10 \\
\hline Boredom & 116 & 43.9 & 1 & 298 & 27.0 & 5 & 41 & 53.9 & 1 \\
\hline Conviviality & 75 & 28.4 & 5 & 572 & 51.9 & 1 & 25 & 32.9 & 5 \\
\hline Increased social control & 2 & 0.8 & 15 & 9 & 0.8 & 15 & 2 & 2.6 & 12 \\
\hline Loss of daily structure & 98 & 37.1 & 3 & 332 & 30.1 & 4 & 33 & 43.4 & 3 \\
\hline \multirow[t]{2}{*}{ Seeing smoking and drinking in movies and series } & 15 & 5.7 & 13 & 25 & 2.3 & 14 & 5 & 6.6 & 11 \\
\hline & \multicolumn{3}{|c|}{ Total N: $264(7.3 \%)$} & \multicolumn{3}{|c|}{ Total $N: 1,102(30.3 \%)$} & \multicolumn{3}{|c|}{ Total N: $76(2.1 \%)$} \\
\hline
\end{tabular}

Descriptive analysis of the motives for smoking more, drinking more, and using more cannabis. $N$ represents the number of respondents using more. \% represents the relative frequency of respondents using more indicating that motive. Rank defines the order of how often the motive was reported, ranging from 1 (highly reported motive) to 15 (lowest reported motive). If an equal number of respondents reported the motive, motives were given the same rank. 
nificant effect on the change in cigarette smoking ( $p=$ 0.015 ), with lower education being associated with more cigarette smoking: participants with a vocational education had about double odds to smoke more compared to those with a higher education $(\mathrm{OR}=2.085 ; p=0.042)$, whereas those having a doctorate were $76 \%$ less likely to smoke more compared to those with a higher education $(\mathrm{OR}=0.245 ; p=0.017)$. Finally, technical unemployed respondents were $64 \%$ more likely to smoke more cigarettes compared to respondents that were tele-working during the lockdown $(p=0.010)$. Gender, living in a city, number of children, and professional occupation were variables without significant effect on the change in smoking cigarettes.

\section{Cannabis Use}

Of the 105 respondents that used cannabis before the lockdown, 26 respondents $(0.7 \%$ of the total sample) stopped using during the lockdown. Of the 3,527 respondents not using cannabis before the lockdown, 31 started using cannabis during the lockdown $(0.9 \%$ of the total sample; $p=0.597)$. Of all respondents, only $2.1 \%$ stated that they used more cannabis than before and only $1.1 \%$ stated to consume less during the lockdown. There was no statistically significant difference between the number of joints per day before and during the lockdown $(0.1 \pm$ 0.5 joints per day, range $0-8$, and $0.1 \pm 0.4$ joints per day, range $0-5 ; p=0.508$ ). As a result, no regression analysis was performed.

\section{Motives for Use of Alcohol, Cigarettes, and Cannabis}

during Lockdown Measures

When observing the motives that drove respondents to consume more cigarettes, alcohol, and cannabis during the COVID-19 measures compared to before, the top reasons were (1) boredom, (2) lack of social contacts, (3) loss of daily structure, (4) reward after a hard-working day, (5) loneliness, and (6) conviviality (see Table 4).

\section{Discussion and Conclusion}

During the COVID-19 lockdown, people self-reported drinking slightly more alcohol compared to before the lockdown, which was negatively associated with age and positively associated with the number of children, unemployment, and homework. The top motive for drinking more was conviviality. The number of cigarettes smoked per day only marginally increased during the lockdown, again with younger respondents having increased odds of smoking more during the lockdown. Similarly, living alone, having a lower educational background, and being technically unemployed increased the odds of smoking more cigarettes during the lockdown as well. The top motive for smoking more cigarettes was boredom.

Cannabis use did not change in times of social isolation. This may indicate a true similarity in the point estimates before and during the lockdown, but it may also reflect a zero net-effect of increased need and reduced availability of cannabis in times of social lockdown (borders are closed, dealers have a harder time to go around unnoticed, and prices increase due to increased demand). Similar to smoking, the top for an increased use of cannabis was boredom. The similarities between ranked motives of cannabis and cigarette smoking are most probably an artefact because most cannabis users are also cigarette smokers. This explains why the ranking of motives is almost the same for smoking cigarettes and cannabis use, and is the result of the fact that no separate questions about motives were asked for the 3 separate substances. It might be an interesting future research question to investigate what drives certain individuals to consume more, less, or equal during a pandemic, and individual assessment of different motives for use, per substance, would here be valuable. Also, focussing on more positive factors might reveal now underreported factors for better resilience and consequently more adequate coping mechanisms than substance use.

This study sample is not optimally representative of the overall Belgian population with 50\% men, less urban people, an average lower education, an estimate of $12 \%$ active in healthcare, and different jobs (possibly with higher technical unemployment rates). In this survey, we mainly note highly educated females (70\%), with $36.8 \%$ being healthcare workers. We found no effect of gender and living in a city on changes in substance use and no effect of educational background for changes in alcohol use. However, our data show that non-healthcare workers were $40 \%$ more likely to consume more alcohol compared to healthcare workers during the lockdown. The respondents from this survey included more healthcare workers as would be expected from a true representative sample, which may infer that, in a true representative population, the differences in alcohol use may be larger than presented here. This needs to be confirmed in larger surveys.

This study has limitations, including the very brief time during which this web-based survey was made accessible (20 days), which yielded a relatively small sample size $(N=3,632)$, while being representative for a uniform period of full lockdown measures. In addition, we did not 
follow-up on the self-reported substance use after this 3-week period, and use may stabilize or increase further with increased duration of lockdown. Another limitation was that we only assessed alcohol, cigarettes, and cannabis and the mean use of alcohol per day, rather than per week, such that individuals consuming only a few drinks per week may have over- or underreported their drinking. This was counterbalanced by the possibility to add "drinking more, or drinking less than usual, or about the same" in the next question regarding their change in alcohol use.

As a whole, these data show that specific groups of individuals, especially younger individuals with lower educational background or those technically unemployed, have a higher risk of increasing their alcohol and/or cigarette use during times of social isolation. It is therefore of importance that care providers and especially general practitioners discuss the use of alcohol, tobacco, and cannabis with their patients, as this often has a beneficial effect on behaviour.

\section{Statement of Ethics}

Ethical approval was obtained. Because this was an anonymous web-based survey, patients were exempt from our Ethical Committee for given written informed consent. Research complied with the appropriate guidelines for human studies and animal welfare regulations and was approved as such by the Medical Ethics Committee of the University Hospital Brussels (UZ Brussel), under reference number 143202043.

\section{Conflict of Interest Statement}

C.L.C. has received lecture fees from Takeda and GME. F.M. received honoraria from Lundbeck, Eli Lilly, and Johnson \& Johnson. None were related with this research. The other authors have nothing to declare.

\section{Funding Sources}

The authors did not receive any funding.

\section{Author Contributions}

N.V. started the project and C.L.C. prepared the 1st draft. S.V.L. performed the analyses. F.M., D.Z., L.S., and S.V.D.A. provided comments and revised the manuscript. All authors were involved in the study setup and approved the final manuscript in its current form.

\section{References}

1 Ahn DG, Shin HJ, Kim MH, Lee S, Kim HS, Myoung J, et al. Current status of epidemiology, diagnosis, therapeutics, and vaccines for novel coronavirus disease 2019 (COVID-19). J Microbiol Biotechnol. 2020;30(3):313-24.

2 Federal Public Service, Health, Food chain safety and Environment [Internet]. Official information of the Belgian government concerning coronavirus Covid-19. What is the government doing? [cited 2020 Apr 19]. Available from: https://www.info-coronavirus.be/en/what-is-the-government-doingabout-it/

3 Usher K, Bhullar N, Jackson D. Life in the pandemic: social isolation and mental health. J Clin Nurs. 2020.
4 Perrin PC, McCabe OL, Everly GS Jr, Links JM. Preparing for an influenza pandemic: mental health considerations. Prehosp Disaster Med. 2009;24(3):223-30.

5 Bansal P, Bingemann TA, Greenhawt M, Mosnaim G, Nanda A, Oppenheimer J, et al. Clinician wellness during the COVID-19 pandemic: extraordinary times and unusual challenges for the allergist/immunologist. J Allergy Clin Immunol Pract. 2020;S22132198(20):30327-5
6 Brooks SK, Dunn R, Amlôt R, Rubin GJ, Greenberg N. A systematic, thematic review of social and occupational factors associated with psychological outcomes in healthcare employees during an infectious disease outbreak. J Occup Environ Med. 2018;60(3):24857.

7 Wu P, Liu X, Fang Y, Fan B, Fuller CJ, Guan $Z$, et al. Alcohol abuse/dependence symptoms among hospital employees exposed to a SARS outbreak. Alcohol Alcohol. 2008;43(6):70612.

8 Beseler CL, Aharonovich E, Hasin DS. The enduring influence of drinking motives on alcohol consumption after fateful trauma. Alcohol Clin Exp Res. 2011;35(5):1004-10. 